\title{
Musicoterapia en Educación: un proyecto de innovación orientado a la inclusión del alumnado con necesidades educativas especiales
}

\author{
Luis del Barrio Aranda (Universidad de Zaragoza, Espanha) \\ delbarri@unizar.es \\ Patricia L. Sabbatella (Universidad de Cádiz, Espanha) \\ patricia.sabbatella@uca.es \\ Melisa Mercadal Brotons (ESMUC, Espanha) \\ brotons@compuserve.com
}

\begin{abstract}
Resumen: Este artículo presenta un proyecto de innovación titulado La práctica de la Musicoterapia: una propuesta educativa de atención a la diversidad del alumnado en Educación Infantil y Primaria realizado en el colegio público de Educación Infantil y Primaria Juan XXIII de Zaragoza (España) entre los años 2012-2017 en el que participaron 32 alumnos con necesidades educativas especiales (NEE) con un musicoterapeuta del centro. Se describe la estructura y se presentan resultados, informando la función de la Musicoterapia en prácticas educativas interdisciplinares y proactivas que avalan su inclusión en contextos educativos.

Palabras clave: Musicoterapia en Educación; Educación Inclusiva; Necesidades Educativas Especiales; Innovación Educativa; Educación Infantil y Primaria.

\section{Music therapy in education: a project of innovation oriented to the inclusion of students with special educational needs}

\begin{abstract}
This article presents an innovation project entitled The Practice of Music Therapy: An Educational Proposal for Attention to the Diversity of the Students in Pre-school and Primary Education in the public school of Pre-school and Primary Education Juan XXIII, Zaragoza (Spain) during the years 2012-2017, involving 32 students with special needs education, with a qualified music therapist. The structure and the results of the project are presented reporting the function of Music Therapy in interdisciplinary and proactive educational practices that guarantee its inclusion in educational contexts.

Keywords: Music Therapy in Education; Inclusive Education; Special Needs Education; Educational Innovation; Pre-school and Primary Education.

\section{Musicoterapia na educação: um projeto de inovação orientado para a inclusão do alunado com necessidades educativas especiais}

Resumo: Este artigo apresenta um projeto inovador intitulado A prática da Musicoterapia: uma proposta educativa para dar atenção à diversidade dos alunos na Educação Infantil e Primária Pública. Este projeto foi realizado na Educación Pré-Escolar e Primária Juan XXIII, em Zaragoza (Espanha) entre 2012 e 2017, envolvendo 32 alunos com necessidades educativas especiais e um musicoterapeuta. O artigo descreve a estrutura da intervenção e apresenta resultados, informando sobre o papel da Musicoterapia nas práticas educativas interdisciplinares e pró-ativas que justificam a sua inclusão no contexto educacional.

Palavras-chave: Musicoterapia na Educação; Ensino Inclusivo; Necessidades Educativas Especiais; Inovação Educacional; Educação Infantil e Primária.

\section{Introducción}

En España, la prevalencia de la tasa de alumnado con necesidades educativas especiales derivadas de una discapacidad matriculado en Enseñanzas de Régimen General ha crecido del 1,97\% al 2,19\% desde el año 1999 al año 2015 (ODISMET, 2016). La Ley Orgánica de Educación (LOE, 2006), establece la normativa para que el alumnado con necesidades educativas especiales (NEE) sea escolarizado en colegios ordinarios haciendo efectiva la filosofía de la educación inclusiva (UNESCO, 1994).

La inclusión educativa del alumnado con necesidades educativas especiales requiere, por parte del profesorado, el desarrollo de propuestas educativas innovadoras 
fundamentadas en resultados de investigación que apoyen su actuación. En este contexto, la Musicoterapia y la Educación Musical aplicadas en el ámbito de las necesidades educativas especiales aportan fundamentos teóricos y metodológicos que convierten a la música en una experiencia psico-educativa integradora de procesos que activan diferentes niveles jerárquicos de percepción, procesamiento y respuesta que involucran experiencias sensoriales, motrices, emocionales, cognitivas y sociales (SABBATELLA, 2014). Siguiendo esta línea de innovación educativa, este artículo presenta el proyecto titulado La práctica de la Musicoterapia: una propuesta educativa de atención a la diversidad del alumnado en Educación Infantil y Primaria (DEL BARRIO, 2012) realizado en el colegio público de Educación Infantil y Primaria Juan XXIII de Zaragoza (España) entre los años 2012-2017.

\section{Musicoterapia y Educación Musical en la atención del alumnado con necesidades edu- cativas especiales}

Los Sistemas Educativos actuales proponen alternativas pedagógico-terapéuticas para la inclusión del alumnado con necesidades educativas especiales tanto en la Educación Infantil y Primaria como en la Educación Especial, siendo una de ellas la música.

La música se utiliza con el alumnado con necesidades educativas especiales de acuerdo con las siguientes modalidades: a) Educación Musical Especial, adapta diferentes métodos pedagógicos musicales para enseñar música al alumnado con algún tipo de discapacidad, b) Educación Musical Inclusiva, adapta los métodos pedagógicos musicales para favorecer el acceso a la educación musical de todos los individuos atendiendo a sus características, intereses, capacidades y necesidades de aprendizaje, y c) Musicoterapia utilizada con un enfoque educativo, que con el objetivo de mejorar la calidad de la educación del alumnado con necesidades educativas especiales, utiliza el sonido y la música para promover la salud física, emocional, intelectual y la comunicación.

La utilización de la Musicoterapia en la Educación Obligatoria y en la Educación Especial presenta diferentes enfoques según la población a la que se dirige (TOMLINSON; DERRINGTON; OLDFIELD, 2012; BUNT, 2003; DAVESON; EDWARDS, 1998; GFELLER, 2000; NORDOFF; ROBBINS, 1982; WILSON, 2001). La Musicoterapia en Educación Especial se enfoca en el desarrollo de habilidades psicoeducativas a fin de promover el desarrollo psicoemocional, social y comunicativo del alumnado (ADAMEK; DARROW, 2005; MCFERRAN; STEPHENSON, 2006; STEPHENSON, 2006). La Musicoterapia en Educación es un área de inserción profesional habitual de los musicoterapeutas, sin embargo, éstos deben seguir demostrando la necesidad de su presencia profesional en colegios de Educación Infantil y Primaria, de Educación Especial y en Equipos de Atención Temprana. Por ello, desde una perspectiva internacional, el nivel de inserción profesional en las diferentes regiones del mundo no es equilibrado.

En España, se han realizado diferentes investigaciones con el objetivo de estudiar el estado de la situación profesional y laboral de los musicoterapeutas (DEL MORAL; MERCADAL-BROTONS; SABBATELLA, 2015; MERCADAL-BROTONS, 2011; ORTIZ; SABBATELLA, 2011; POCH, 2008a, 2008b; SABBATELLA; MERCADAL-BROTONS, 2014). Estos estudios informan que los musicoterapeutas trabajan y/o desarrollan proyectos para promover la inclusión de la musicoterapia en el área de la Educación Obligatoria y de la Educación Especial. Específicamente, el estudio de Poch enumera las instituciones públicas y privadas donde se realiza Musicoterapia, incluyendo las instituciones educativas y organiza la información por Comunidades Autónomas (POCH, 2008a; 2008b). 
La Ley Orgánica 1/1990, de 3 de octubre, Boletín Oficial del Estado (BOE) de 4 de octubre, de Ordenación General del Sistema Educativo (LOGSE), reguló la enseñanza de la música en los diferentes niveles de las enseñanzas de régimen general ${ }^{1}$ (Educación Infantil, Primaria y Secundaria) y las enseñanzas de régimen especial (Bachillerato Artístico Musical, escuelas de música y conservatorio). Esta ley potenció la profesionalización de la Educación Musical equiparando el nivel académico de los estudios de música a rango universitario Real Decreto (RD) 1542/1994, de 8 de julio, (BOE de 9 de agosto). Para impartir las enseñanzas musicales en la etapa de Educación Primaria se creó la figura profesional del Maestro especialista de Educación Musical (LOGSE, art. 16). Para formar estos profesionales se crearon los estudios universitarios de Maestro especialista en Educación Musical (RD 1440/1991, de 30 de agosto), que se establece el título universitario oficial de Maestro en sus diversas especialidades y las directrices generales propias de sus planes de estudios (BOE de 11 de octubre). En su estructura curricular, la organización de los planes de estudios de Maestro posibilitó la inclusión de asignaturas optativas para completar la formación del alumnado, pudiéndose ofrecer asignaturas de Musicoterapia, como complemento de formación, en los estudios de Maestro Especialista en Educación Musical, Maestro de Educación Especial y Maestro de Audición y Lenguaje. De un total de 69 Universidades Públicas y Privadas, 17 ofrecieron asignaturas de Musicoterapia en los estudios de Maestro entre los cursos académico 1993/1994 hasta el 2004/2005 (SABBATELLA, 2005).

Aunque la enseñanza de la música en España se vio favorecida por la LOGSE, no se contempló la inclusión del Maestro Especialista en Educación Musical en la Educación Especial. Las modificaciones en las posteriores leyes educativas, Ley Orgánica 2/2006, de 3 de mayo, de Educación (LOE) aprobada el 6 de abril de 2006 con vigencia hasta el 2013 y la actual Ley Orgánica 8/2013, de 9 de diciembre, para la mejora de la calidad educativa (LOMCE) tampoco incluyeron la presencia del Maestro de Educación Musical en Educación Especial, recayendo tal decisión en las distintas administraciones autonómicas.

\section{Musicoterapia e innovación educativa en la comunidad de Aragón}

La tasa de alumnado con NEE derivadas de una discapacidad matriculado en Enseñanza de Régimen General de la comunidad de Aragón es una de las más bajas entre las comunidades autónomas de España (1,83\%) (ODISMET, 2016). Como una medida educativa de promoción de la inclusión, la administración educativa de esta comunidad aprueba la matriculación del alumnado con NEE en los colegios ordinarios de Educación Infantil y Primaria. Para atender al alumnado con NEE, cada colegio propone un Plan de Atención a la Diversidad (PAD) que recoge el conjunto de actuaciones educativas a desarrollar durante un curso escolar desde las diferentes áreas de actuación.

En el apartado anterior se fundamentó el uso de la Musicoterapia en contextos educativos y con alumnado con discapacidad, argumentos que apoyan su integración en el Plan Atención a la Diversidad de los Proyectos Educativos de los colegios ordinarios ${ }^{2}$. La idea de presentar este proyecto de innovación surge por la preocupación por mejorar la atención educativa del alumnado con NEE y por el interés personal del Maestro de Educación Musical del centro que también es Musicoterapeuta ${ }^{3}$. Con la finalidad de integrar la Musicoterapia en el Proyecto Educativo de Centro se presentó en el año 2012, el proyecto de innovación La práctica de la Musicoterapia: una propuesta educativa de atención a la diversidad del alumnado en Educación Infantil y Primaria, aprobado por el Departamento de Educación del Gobierno de Aragón y desarrollado entre los años 2012-2017 en el colegio público de Educación Infantil y Primaria Juan XXIII de Zaragoza (DEL BARRIO, 2012). 


\section{Descripción del proyecto de innovación de musicoterapia en educacion}

El proyecto de innovación de Musicoterapia se propone en el Plan de Atención a la Diversidad como un servicio para favorecer la atención educativa del alumnado con NEE dentro del horario lectivo. El objetivo general del proyecto es implementar la Musicoterapia en la atención del alumnado con NEE escolarizado en los niveles de Educación Infantil y Primaria y valorar su contribución a la educación inclusiva.

Las fases del proyecto son las siguientes:

a. Selección de participantes. Se realiza una reunión con el equipo multidisciplinar (Especialista de Pedagogía Terapéutica, Logopeda, Maestra Tutora, Maestro de Educación Musical-Musicoterapeuta) en la que se revisa el informe psicopedagógico y el historial académico de cada estudiante con NEE, las características y las necesidades particulares de cada alumno/a y se deciden qué alumnos participan en el proyecto de Musicoterapia.

b. Plan de trabajo. Se analizan las necesidades de cada uno de los participantes para establecer el plan de trabajo en Musicoterapia individual y grupal. El estudio de la documentación personal, la evaluación inicial y las primeras sesiones de musicoterapia aportan información sobre las necesidades de cada participante. La entrevista familiar y la reunión con la maestra tutora amplían esa información con datos referidos al comportamiento del niño/a en diferentes situaciones y contextos.

c. Evaluación. La observación participante es la técnica de recogida de información utilizada en las sesiones de Musicoterapia. Finalizada cada sesión se cumplimenta un cuaderno de campo donde se recogen los logros, las dificultades y los comportamientos del alumno y otros datos significativos para analizar la intervención del niño y la actuación del Musicoterapeuta en el proceso de Musicoterapia. Además, la psicóloga del centro utiliza el Trail Making Test (TMT-A) y (TMT-B) para valorar la atención focalizada y dividida en el alumnado con Discapacidad Intelectual y la Escala de Inteligencia Wechsler (WISC-R) para valorar este aspecto en el alumnado con Retraso General del Desarrollo. El seguimiento del trabajo en Musicoterapia requiere un protocolo de reuniones quincenales con las maestras tutoras y mensuales con el resto de profesionales del Equipo de Orientación que trabajan con el alumno. Las reuniones mensuales con la familia informan de la evolución del niño y permiten recoger información para el trabajo en las sesiones relacionada con: los comportamientos y las actitudes musicales del niño en el hogar; las obras musicales que forman parte de la historia musical del niño para incorporarlas en las sesiones; los comportamientos y las actitudes del niño en el hogar y los diferentes modos de actuación de la familia en momentos de crisis.

\section{Participantes}

El número de alumnos matriculados en el colegio es de 448 alumnos. Durante la implantación del proyecto (2012-2017) han participado 32 alumnos en total (7,1\%), de los cuales 19 fueron alumnos de Educación Infantil (59,4\%) y 13 de primer ciclo de Primaria (40,6\%), con edades comprendidas entre los 3 y 8 años. Según el género, 28 fueron niños $(87,5 \%)$ y 4 niñas $(12,5 \%)$. El alumnado participante presentaba los siguientes diagnósticos (Cuadro 1): 
DEL BARRIO, L. ; SABBATELLA, P. L.; BROTONS, M. M. Musicoterapia en Educación: un proyecto de innovación orientado a la inclusión del alumnado con necesidades educativas especiales Revista Música Hodie, 2019, v.19: e51723

Cuadro 1: Clasificación de los participantes según el diagnóstico y el género (Del Barrio; Sabbatella; Mercadal-Brotons, 2018).

\begin{tabular}{|c|c|c|c|c|}
\hline Diagnóstico & Niños & Niñas & Total & Porcentaje \\
\hline Discapacidad Intelectual & 10 & 2 & 12 & $37,5 \%$ \\
\hline Trastorno Espectro Autista & 8 & - & 8 & $25 \%$ \\
\hline Discapacidad física & 4 & - & 4 & $12,5 \%$ \\
\hline Trastorno Déficit por Atención con Hiperactividad & 4 & - & 4 & $12,5 \%$ \\
\hline Trastorno Grave de Conducta & 2 & - & 2 & $6,2 \%$ \\
\hline Retraso Global del Desarrollo & - & 2 & 2 & $6,2 \%$ \\
\hline TOTAL & $\mathbf{2 8}$ & $\mathbf{4}$ & $\mathbf{3 2}$ & $\mathbf{1 0 0}$ \\
\hline
\end{tabular}

Se determinan los siguientes ámbitos de intervención, y la concreción de los objetivos psicoeducativos y contenidos para cada caso (Cuadro 2).

Cuadro 2: Ámbitos de intervención, objetivos psicoeducativos y contenidos (Del Barrio; Sabbatella; Mercadal-Brotons, 2018)

\begin{tabular}{|c|c|}
\hline ÁMBITOS & OBJETIVOS PSICOEDUCATIVOS Y CONTENIDOS \\
\hline \multirow{5}{*}{ MOTOR } & Conciencia corporal (imagen, esquema y concepción corporal) \\
\hline & Desarrollo sensoriomotor (actividad motora y postural) \\
\hline & Desarrollo psicomotor (equilibrio estático y dinámico) \\
\hline & Motricidad gruesa (agilidad, tonicidad, velocidad y control) \\
\hline & Motricidad fina (coordinación óculo manual) \\
\hline \multirow{2}{*}{ EMOCIONAL } & Conocimiento, comprensión y gestión de las emociones \\
\hline & Iniciativa para la realización de tareas \\
\hline \multirow{3}{*}{ COGNITIVO } & Habilidades sensoriales y perceptivas \\
\hline & Atención, concentración y memoria \\
\hline & Autoestima, autoconfianza y autonomía \\
\hline \multirow{3}{*}{ COMUNICATIVO } & Conciencia fonológica (fonación, articulación y resonancia) \\
\hline & Habilidad comunicativa verbal y no verbal \\
\hline & Aspecto expresivo del lenguaje verbal \\
\hline \multirow[b]{2}{*}{ SOCIAL } & Estilo de comportamiento social (participativo, dominante, pasivo) \\
\hline & $\begin{array}{l}\text { Destrezas socioemocionales (empatía, responsabilidad, cooperación, } \\
\text { respeto, asertividad) }\end{array}$ \\
\hline \multirow{8}{*}{ MUSICAL } & Gustos y preferencias musicales \\
\hline & $\begin{array}{l}\text { Elementos rítmicos (patrones predominantes, motivos, secuencias, uso } \\
\text { del silencio) }\end{array}$ \\
\hline & Elementos melódico-tonales (intervalos, motivos, melodías predominantes) \\
\hline & Estructura y forma musical (repetición, organización de ideas musicales) \\
\hline & $\begin{array}{l}\text { Habilidades vocales (vocalizaciones, entonación, articulación, } \\
\text { intencionalidad, expresión) }\end{array}$ \\
\hline & Habilidades instrumentales (manipulación, técnica, expresión) \\
\hline & Memoria musical (repetición, medio largo y largo plazo) \\
\hline & Creatividad (habilidad de transformación, improvisación, composición) \\
\hline
\end{tabular}




\section{Metodología de Trabajo}

Las sesiones de Musicoterapia se desarrollan desde el mes de octubre al mes de junio de cada uno de los cursos escolares comprendidos desde el curso 2012/13 al curso 2016/17. Dentro del horario lectivo de clase tienen una frecuencia semanal, con una duración de 40 minutos. La autorización de las familias y de la maestra tutora permiten la atención del niño en las sesiones de musicoterapia durante el horario lectivo. Las sesiones pueden ser individuales, en pareja, en pequeño grupo o con el grupo-clase en función de las necesidades de cada caso.

La estructura de la sesión puede variar de acuerdo con las necesidades de los participantes. Desde un punto de vista metodológico, el musicoterapeuta observa y adapta su intervención a las necesidades y los objetivos integrando las producciones del alumnado en el desarrollo de actividades. La participación activa es un principio básico en el enfoque implementado, independientemente de las limitaciones o dificultades del alumnado.

En función de los objetivos se utilizan diferentes técnicas de Musicoterapia y de Educación Musical, entre las que se encuentran: tocar instrumentos musicales; improvisación instrumental y vocal; creación de canciones; canto y expresión vocal; interacción músicamovimiento; escucha y audición musical activa.

$\checkmark$ Tocar instrumentos musicales integra la motricidad fina y la coordinación al ajustar la habilidad manual a las características del instrumento musical. Estimula la capacidad auditiva y la memoria. Favorece la expresión.

$\diamond$ La improvisación instrumental y/o vocal como técnica interactiva y expresiva promueve la capacidad auditiva, la interacción y la comunicación no verbal.

$\diamond$ La creación o composición de canciones facilita el desarrollo expresivo musical y lingüístico, la memoria y la organización de ideas musicales. El producto creativo musical resulta significativo para el alumno al relacionarlo con momentos, situaciones, personas o hechos de su vida.

$\checkmark$ El canto y la expresión vocal estimulan la memoria fonológica y lingüística, la expresión fonológica, la articulación, la respiración, el fraseo y el desarrollo del lenguaje. Promueve el contacto con los recursos expresivos de la voz y su conexión con las emociones.

$\diamond$ La interacción música-movimiento permite trabajar la conciencia corporal (la imagen corporal, el esquema corporal y la concepción corporal), la interacción corporal en el espacio, con los objetos y con los compañeros, así como el fortalecimiento físico y el desarrollo socioafectivo.

$\checkmark$ La escucha y la audición musical activa promueven el reconocimiento de sonidos y la memoria sonora y musical, integrando el trabajo perceptivo a través de la sensorialidad, la sensibilidad auditiva y la escucha estimulando la atención tanto auditiva como cognitiva.

\section{Resultados}

Los resultados describen, de manera global, la evolución de los casos tratados $(\mathrm{N}=32)$ durante los cinco años de implementación del proyecto, en el que no todos los niños participaron a lo largo del período completo. Las técnicas de recogida de datos fueron: la observación participante, el cuaderno de campo con el análisis descriptivo de las sesiones, los resultados de los diferentes tests de evaluación de la atención Trail Making Test (TMT-A y TMT-B) y de la Escala de Inteligencia Wechsler (WISC-R) y la información de las actas 
de las reuniones semanales con la maestra tutora de cada curso, las actas de las reuniones quincenales con los profesionales del Equipo de Orientación Psicopedagógica y de las reuniones mensuales con las familias de los participantes. Los resultados se presentan en función de las poblaciones participantes y la relevancia de los ámbitos trabajados según las necesidades educativas particulares.

\section{Discapacidad Intelectual (DI)}

Participaron 12 alumnos (37,5\%) con distintos niveles de gravedad (8 discapacidad intelectual leve, 3 discapacidad moderada y 1 discapacidad severa).

En el ámbito motor, 9 participantes (75\%) mejoraron la conciencia corporal y el desarrollo psicomotor en actividades estáticas, la motricidad gruesa y de movimiento en la interacción con el espacio, los objetos y los compañeros. En el ámbito emocional, 10 alumnos $(83,3 \%)$ demostraron una mejora en la motivación y la iniciativa para tomar decisiones en las actividades musicales con diferentes grados de exigencia. En el ámbito cognitivo, 11 alumnos $(91,6 \%)$ mejoraron la atención, el contacto visual y el turno de participación; 5 alumnos (41\%) la autoconfianza, la autoestima y la autonomía en las actividades creativas. En cuanto al ámbito comunicativo, 8 participantes $(66,6 \%)$ mejoraron la conciencia y la memoria fonológica y el incremento de la dimensión expresiva del lenguaje. En el ámbito social, la totalidad de los participantes mejoraron sus habilidades de relación y convivencia con los demás compañeros. En el ámbito musical, 8 alumnos (66,6\%) mejoraron la entonación, la intencionalidad y el desarrollo de la dimensión expresiva vocal.

\section{Trastorno del Espectro Autista (TEA)}

Participaron 8 alumnos (25\%) con edades comprendidas entre 3 y 8 años.

En el ámbito motor, todos los participantes mejoraron el control y la regulación de las estereotipias, la conciencia corporal, la actividad motora gruesa y el control postural, el equilibrio dinámico, logrando un equilibrio estático más duradero, y solo 5 alumnos $(62,5 \%)$ la coordinación motriz y la coordinación óculo manual. En el ámbito emocional, 5 alumnos $(62,5 \%)$ mejoraron la comprensión y la expresión de emociones asociadas a la visualización de imágenes y 4 (50\%) fueron capaces de describir sus propias emociones. En el ámbito cognitivo, 6 participantes (75\%) mejoraron su atención, concentración y memoria y el turno de participación al tocar instrumentos musicales. En el ámbito comunicativo, 5 participantes $(62,5 \%)$ mejoraron las habilidades comunicativas verbales integrando el uso de nexos gramaticales en la producción de textos entonados con intención comunicativa, mejorando la funcionalidad expresiva del lenguaje. En el ámbito social, 5 participantes $(62,5 \%)$ mejoraron su relación con los compañeros desarrollando un comportamiento social más participativo, mejorando la escucha, el respeto y la responsabilidad. En el ámbito musical, 6 alumnos (75\%) desarrollaron sus habilidades instrumentales mejorando la precisión, la velocidad interpretativa y la memoria musical; 5 (62,5\%) mejoraron la entonación y la expresión vocal en la interpretación de canciones y 6 (75\%) potenciaron su habilidad creativa musical. El total de los alumnos, 8 (100\%) mejoraron la habilidad comunicativa no verbal asociada a la práctica instrumental. 


\section{Discapacidad física}

Participaron 4 alumnos (12,5\%) con discapacidad física de diferente origen: 2 con discapacidad motora y deficiencia visual, 1 con hipotonía muscular en la mano izquierda y 1 con lesión cerebral que afecta al desarrollo psicomotor y al lenguaje (apraxia).

En el ámbito motor, 3 alumnos (75\%) mejoraron la conciencia corporal, la actividad motora y postural y el equilibrio dinámico con un avance moderado en el equilibrio estático. Los 2 alumnos (50\%) con mayor control motor mejoraron la agilidad y la coordinación motriz. En el ámbito emocional, 3 alumnos (75\%) mejoraron la iniciativa y reconocieron el beneficio de trabajar sus limitaciones físicas. En el ámbito cognitivo, 3 participantes (75\%) mejoraron la autoestima y la autoconfianza. La superación de las dificultades relacionadas con las tareas motrices les permitió afrontar con naturalidad y decisión cada nuevo reto. En el ámbito comunicativo, el alumno con apraxia del habla (25\%) mejoró la memoria fonológica, la articulación de fonemas y el lenguaje funcional. La gestualidad corporal y la interpretación de canciones favorecieron el desarrollo de las habilidades comunicativas verbales y no verbales en todos los participantes. En el ámbito social, 3 alumnos (75\%) mejoraron el modo de relacionarse con los compañeros al demostrar un comportamiento más participativo. En el ámbito musical, 3 participantes (75\%) mejoraron la habilidad de tocar instrumentos tanto en la manipulación como en la agilidad en la producción y la reproducción de ritmos y melodías.

\section{Trastorno Déficit por Atención con Hiperactividad (TDAH)}

Participaron 4 alumnos (12,5\%).

En el ámbito motor los 4 participantes (100\%) redujeron la impulsividad con una mejora de la actividad motora y postural, equilibrio dinámico y habilidades como la tonicidad, la agilidad y la velocidad en actividades de movimiento y percusión. Por su parte, 2 alumnos (50\%) experimentaron una mejora del equilibrio estático y de la motricidad fina en el manejo de los instrumentos. En el ámbito emocional, 3 alumnos (75\%) mejoraron la observación y la identificación de los sentimientos de los otros y la comprensión de las propias emociones y mejoraron la iniciativa en las actividades de música y movimiento. En el ámbito cognitivo, 3 alumnos (75\%) mejoraron la atención, la concentración y la memoria en actividades musicales en general y en las que requieren implican dos acciones simultáneas para su realización. En el ámbito comunicativo, 2 alumnos (50\%) mejoraron la habilidad para respetar el turno de palabra, el turno en la improvisación musical, mirar a los ojos de quien habla y no cambiar de tema al conversar.

En el ámbito social, 3 alumnos (75\%) mostraron capacidad para tomar decisiones y comportarse de manera adecuada en distintas situaciones, desarrollando habilidades de cooperación para realizar actividades con otros compañeros. En el ámbito musical, 2 alumnos (50\%) demostraron una mejora en el desarrollo de habilidades relacionadas con la interpretación de instrumentos de percusión y en la capacidad creativa musical tanto en la transformación del texto de canciones como en la improvisación instrumental de motivos e ideas musicales.

\section{Trastorno grave de conducta (TGC)}

Participaron 2 alumnos $(6,2 \%)$ con (TGC).

En el ámbito motor, los 2 participantes (100\%) redujeron los momentos de impulsividad, mejoraron la conciencia corporal, la actividad motora y postural, el equilibrio, 
la expresión motriz gruesa de agilidad, tonicidad muscular y la velocidad. En el ámbito emocional, los 2 participantes (100\%) mejoraron la identificación de las emociones de los otros, el conocimiento y la comprensión de las emociones propias. En el ámbito cognitivo, 1 alumno (50\%) mejoró la atención y la autoconfianza en el desempeño de las actividades musicales. En el ámbito comunicativo, los 2 alumnos (100\%) mejoraron las habilidades de comunicación no verbal en la actividad creativa musical y en la habilidad expresiva verbal. En el ámbito social, los 2 alumnos (100\%) redujeron la intensidad y la cantidad de conductas sociales no adaptadas mostrando un comportamiento social más participativo adaptándose a las normas del grupo al realizar las actividades musicales, respeto, empatía, responsabilidad, capacidad de esperar turnos, de compartir instrumentos musicales, de focalizar la atención y el contacto visual. En el ámbito musical, los 2 participantes (100\%) mejoraron las habilidades para tocar instrumentos de percusión y en la creación de ideas musicales. Un participante (50\%) mejoró la entonación y la expresión vocal tanto en la interpretación como en la composición de canciones.

\section{Retraso global del desarrollo (RGD)}

Participaron 2 alumnas $(6,2 \%)$ con Retraso Global del Desarrollo. Las áreas afectadas de ambas participantes fueron el neurodesarrollo motriz y lingüístico.

En el ámbito motor, las 2 participantes (100\%) mejoraron la imagen corporal, la actividad motora y postural, el equilibrio dinámico, la agilidad, la velocidad y la respuesta motriz sincronizada con la música. Una de ellas (50\%) la resistencia que afectó a la mejora del control corporal (tensión-relajación). En el ámbito cognitivo, las 2 alumnas (100\%) mejoraron las habilidades sensoriales y perceptivas motoras, la atención, la capacidad de reacción y la memoria inmediata para adaptar el movimiento al sonido y la música. En el ámbito comunicativo, las 2 alumnas (100\%) mejoraron la conciencia y la memoria fonológica y la articulación consonántica. Una de ellas (50\%) aumentó el vocabulario debido al aprendizaje de canciones. En el ámbito social, las 2 alumnas (100\%) mejoraron el respeto, la empatía y la cooperación mostrando un estilo de comportamiento social participativo en los distintos grupos de trabajo. En el ámbito musical, las 2 participantes (100\%) mejoraron la memoria musical y la habilidad creativa, mientras que 1 alumna (50\%) mejoró su habilidad vocal en la interpretación de canciones.

\section{Conclusiones y discusión}

Como se ha indicado, adaptar el curriculum educativo a las necesidades del alumnado requiere implementar líneas de innovación entre las que se encuentra la Musicoterapia; los resultados de este proyecto respaldan su inclusión en contextos educativos.

Respecto al proyecto de innovación y su inclusión en el contexto del centro educativo conviene destacar su repercusión en la organización diaria de la actividad del colegio en referencia a la gestión y la organización de los aspectos administrativos y educativos. A lo largo de cinco años, la inclusión de las actividades de Musicoterapia en el horario lectivo supuso poner en práctica, de forma natural, una actividad no contemplada en el curriculum educativo destinada a atender las necesidades del alumnado con NEE participante. Si nos referimos a la repercusión en el equipo interdisciplinar, la información recabada en las sesiones de Musicoterapia y su discusión con el equipo docente proporcionó un mayor conocimiento de las destrezas de los alumnos en un contexto musical y no musical. Durante el curso, el Equipo de Orientación recomendó posibles candidatos para participar en el 
proyecto de Musicoterapia. El Equipo Directivo facilitó los recursos materiales, espaciales y temporales necesarios para el proyecto. El Servicio de Inspección y los representantes de la Administración Educativa también mostraron interés hacia el proyecto, lo que determinó que el colegio fuera propuesto como centro de atención educativa preferente para el alumnado con Trastorno Espectro Autista (TEA).

El trabajo con las familias del alumnado participante permitió obtener información sobre el comportamiento en contextos cotidianos y sociales diferentes al entorno educativo, contribuyó al establecimiento de pautas de intervención para la reducción de conductas negativistas y promovió el establecimiento y el seguimiento de estrategias reflexivas de autoinstrucción, orientadas a la autorregulación física y la gestión emocional en los momentos de crisis. La colaboración de las familias con el musicoterapeuta motivó la implementación de actividades complementarias en el hogar.

Desde el punto de vista de la intervención, la Musicoterapia en Educación se centra en el individuo y en la formación humana integral y no contempla el desarrollo de objetivos musicales. Sin embargo, el trabajo de las habilidades vocales e instrumentales, inherente a la propia experiencia musical, favoreció la sensibilización musical, el entrenamiento de la escucha y la comprensión sonora, la respuesta imitativa y creativa, la memoria musical, la articulación, la entonación y la expresión vocal, la habilidad psicomotriz de la percusión que implica el trabajo de instrumentos corporales diversos, el conocimiento y el manejo de los instrumentos de pequeña percusión, la iniciación a la técnica instrumental, la exploración sonora y la creatividad en sus diversas formas de expresión. En el alumnado participante, en el ámbito motor, la interacción entre la música, el movimiento y la expresión integró de un modo natural e intuitivo, el trabajo sobre la conciencia corporal, la actividad motora y postural, el equilibrio y la expresión motriz. Como consecuencia, en el ámbito emocional se promovió la expresión sonora y gestual a través de la imitación corporal, vocal e instrumental favoreciendo la empatía y la afectividad. En este contexto, la improvisación musical favoreció el desarrollo de habilidades socioemocionales de comunicación no verbal. En el ámbito cognitivo y social, la experiencia compartida, la habilidad exploratoria y creativa y la autonomía se vieron reforzadas en el trabajo en entornos educativos proactivos y colectivos. El canto colectivo facilitó la interacción interpersonal con el intercambio de ideas que animaron el diálogo, la creatividad y la intencionalidad comunicativa. La dimensión comunicativa no verbal de la experiencia musical potenció la intención comunicativa del alumnado con dificultades expresivas del lenguaje. La improvisación ofreció un modo alternativo de comunicación no verbal que estimuló la exploración, la iniciativa, la intención y la expresión. De un modo general, el canto favoreció el trabajo prosódico del habla, la dicción, la memoria fonológica y lingüística, contribuyendo, en un sentido amplio, a la mejora de la dimensión expresiva del lenguaje.

La integración de la Musicoterapia en el Proyecto Educativo de Centro y en el Plan de Atención a la Diversidad proporciona un servicio complementario que atiende las dificultades del alumnado con NEE, complementa el trabajo del equipo profesional y contribuye a la construcción de un modelo educativo integral e interdisciplinar. Este enfoque conlleva pautas comunes de intervención que refuerzan la cohesión educativa en el trabajo de las mismas dificultades. Para ello, el musicoterapeuta necesita conocer las características propias del alumnado con NEE y las competencias profesionales específicas que le permiten su actuación en contextos educativos.

La evaluación institucional del proyecto por parte de los representantes de la Administración Educativa, el Equipo Directivo del colegio y el profesorado implicado, determinó la consolidación de la Musicoterapia como una propuesta de mejora en la 
atención del alumnado con NEE. Desde el año 2012 hasta la actualidad, la puesta en marcha de este proyecto de innovación pionero en la comunidad de Aragón: La práctica de la Musicoterapia: una propuesta educativa de atención a la diversidad del alumnado en Educación Infantil y Primaria, impulsó la integración de la Musicoterapia como actividad profesional en el Proyecto Educativo del Centro de Educación Infantil y Primaria Juan XXIII de Zaragoza (España). Su continuidad y repercusión sentaron las bases de su consolidación.

En la escuela del siglo XXI, la dimensión humana de la educación demanda la atención y el trabajo de las habilidades del alumnado con y sin discapacidad. Su desarrollo requiere la integración de medidas de actuación complementarias desde las cuales enfocar las acciones hacia prácticas educativas proactivas, dinámicas y funcionales como medida de promoción de la educación inclusiva. La Musicoterapia es una disciplina que contribuye a ello.

\section{Notas}

1 Las enseñanzas de régimen general se corresponden con la educación obligatoria

2 En España, al día de la fecha, no se incluye la Musicoterapia como área especializada en el organigrama de los centros educativos.

3 Desde el año 2006 la Universidad de Zaragoza oferta formación de postgrado en el nivel de Master en Musicoterapia en el que se han formado 46 Musicoterapeutas hasta el año 2017.

\section{Referencias}

ADAMEK, Mary, S.; DARROW, Alice-Ann. Music in Special Education. Silver Spring, MD: American Music Therapy Association, 2005.

BUNT, Leslie. Music Therapy with Children: A complementary Service to Music Education? British Journal of Music Education, v. 20, n. 2, p. 179-195, 2003.

DAVESON, Barbara; EDWARDS, Jane. A Role for Music Therapy in Special Education. International Journal of Disability, Development and Education, v. 45, n. 4, p. 449-455, 1998.

DEL BARRIO, Luis. La práctica de la Musicoterapia: una propuesta educativa de atención a la diversidad del alumnado en Educación Infantil y Primaria. Proyecto de innovación. Departamento de Educación, Universidad, Cultura y Deporte del Gobierno de Aragón, 2012. < http://www. redined.mec.es >. 00220111000140.

DEL MORAL, María Teresa; MERCADAL-BROTONS, Melissa; SABBATELLA, Patricia. Un Estudio Descriptivo sobre el Perfil del Musicoterapeuta en España. Música, Terapia y Comunicación, 35, p. 15-29, 2015.

GFELLER, Kate. Musicoterapia en el Entorno Educativo, en Davis, William; Gfeller, Kate y Thauth, Michael. Introducción a la Musicoterapia: Teoría y Práctica, Barcelona: Boileau, 2000.

LOGSE, Ley Orgánica 1/1990, de 3 de octubre, de Ordenación General del Sistema Educativo. (BOE de 4 de octubre), 1990.

LOE, Ley Orgánica 2/2006, de 3 de mayo, de Educación. (BOE de 4 de mayo), 2006.

LOMCE, Ley Orgánica 8/2013, de 9 de diciembre, para la mejora de la calidad educativa. (BOE de 10 de diciembre), 2013.

McFERRAN, Katrina; STEPHENSON, Jennifer. Music Therapy in Special Education: Do we need more evidence? British Journal of Music Therapy, v. 20, n. 2, p. 121-128, 2006. NORDOFF, Paul; ROBBINS, Clive. Music Therapy in Special Education. San Luis: MMB Music, 1982. 
ODISMET. Tasa de alumnado con necesidades educativas especiales derivadas de una discapacidad matriculado en Enseñanzas de Régimen General. Fundación ONCE, 2016. < http:// www.odismet.es/es/datos/3educacin-y-formacin-profesional>.

ORTIZ, Francisco P; SABBATELLA, Patricia L. Perfil profesional del Musicoterapia en España: Resultados de investigación. En : Sabbatella, P. (Coord.). IDENTIDAD Y DESARROLLO PROFESIONAL DEL MUSICOTERAPEUTA EN ESPAÑA. ACTAS DEL III CONGRESO NACIONAL DE MUSICOTERAPIA, 2010. Granada: Grupo Editorial Universitario, 2011. p. 49-54.

POCH, Serafina. Rol del Profesional Musicoterapeuta. ACTAS DEL II CONGRESO NACIONAL DE MUSICOTERAPIA. FORMACIÓN, PROFESIONALIZACIÓN E INVESTIGACIÓN. Zaragoza: Estudio de Palabras, 2008a. p. 54-57,

. Los Profesionales Musicoterapeutas en España en la Actualidad. ACTAS DEL II CONGRESO NACIONAL DE MUSICOTERAPIA. FORMACIÓN, PROFESIONALIZACIÓN E INVESTIGACIÓN. Zaragoza: Estudio de Palabras, 2008b. p. 54-57.

SABATELLA, Patricia L. Music Therapy Training within the European Higher Education System: A Survey on Music Therapy Training Courses in Spain. In: Aldridge, D.; Fachner, J. \& Erkkilä, J. (Ed.). MANY FACES OF MUSIC THERAPY - PROCEEDINGS OF THE 6TH EUROPEAN MUSIC THERAPY CONGRESS. Jyväskylä, Finland, Music Therapy Today, v. 6, Issue 4, Nov. 2005, p. 1378-1411. Retrieved from: <http://www.wfmt.info/Musictherapyworld/modules/archive/stuff/ reports/Proceedings_Screen.pdf\#page $=25>$.

. Atención a la diversidad en Educación Musical: Orientaciones teóricas y metodológicas para su aplicación en el aula. En J. L. Aróstegui (Ed.), La música en Educación Primaria. Madrid: Dairea, 2014.

SABBATELLA, Patricia L; MERCADAL-BROTONS, Melissa. Perfil profesional y laboral de los musicoterapeutas españoles: Un estudio descriptivo. Revista Brasileira de Musicoterapia, 17, p. 6-16, 2014.

STEPHENSON, Jennifer. Music Therapy and the Education of Students with Severe Disabilities. Education and Training in Developmental Disabilities, v. 41, n. 3, p. 290-299, 2006.

TOMLINSON, Jo; DERRINGTON, Philippa; OLDFIELD, Amelia. Music Therapy in Schools: Working with Children of All Ages in Mainstream and Special Education. London: Jessica Kingsley, 2012.

UNESCO. Informe Final. Conferencia mundial sobre necesidades educativas especiales: acceso y calidad. Madrid: UNESCO/Ministerio de Educación y Ciencia, 1994.

WILSON, Brian L. Models of Music Therapy Intervention in School Settings. Silver Spring, MD: American Music Therapy Association, 2001.

Luis del Barrio es PhD. Licenciado en Pedagogía Musical por el Conservatorio Superior de Aragón (Espanha) (2005). Máster en Musicoterapia por la Universidad de Zaragoza (2008). Doctor en Ciencias de la Educación y Pedagogía Musical por la Universidad de Barcelona (2012). Director y profesor en Máster en Musicoterapia de la Universidad de Zaragoza (2015). Actualmente es Profesor Asociado en la Universidad de Zaragoza desde 2009, Maestro de Educación Musical (1997) y Musicoterapeuta por la Asociación Española de Musicoterapeutas Profesionales (AEMT). Miembro del grupo de Investigación HUM-794: Plan Andaluz de Investigación, Desarrollo e Innovación. Experiencia profesional e investigación musical y en musicoterapia en el área Educativa. 
Patricia L. Sabbatella es PhD, EMTR-Supervisor, SMTAE. Profesora Titular Universidad de Cádiz (Espanha) y Musicoterapeuta. Directora del grupo de Investigación HUM-794: Plan Andaluz de Investigación, Desarrollo e Innovación. Experiencia profesional y de investigación en las áreas Educativa y Clínica con especial interés en la evaluación musicoterapéutica. Participa activamente en la WFMT, EMTC y Asociación Española de Musicoterapeutas Profesionales (AEMP).

Melissa Mercadal Brotons es PhD, MT-BC, SMTAE. Psicóloga por la Universidad de Barcelona y Máster en Musicoterapia por la Florida State University. Directora de la Escola Superior de Música de Catalunya (ESMUC), profesora del Departamento de Educación Musical y directora del programa de Máster en Musicoterapia (ESMUC). Presidente de la World Federation of Music Therapy (WFMT) y Delegada Española de la European Music Therapy Confederation (EMTC). Ha publicado extensivamente a nivel nacional e internacional trabajos de investigación en el campo de la geriatría, demencias y discapacidad. Miembro del grupo de Investigación: “Música y Creación” (ESMUC). 